\title{
Ortho Arylation of Acetanilides via Pd(II)-Catalyzed C-H Functionalization
}

\author{
Shangdong Yang, Bijie Li, Xiaobing Wan, and Zhangjie Shi* \\ Beijing National Laboratory of Molecular Sciences (BNLMS) and Key Laboratory of \\ Bioorganic Chemistry and Molecular Engineering of Ministry of Education, College \\ of Chemistry and Green Chemistry Center, Peking University, Beijing 100871 and \\ State Key Laboratory of Organometallic Chemistry, Chinese Academy of Sciences, \\ Shanghai 200032, China
}

\section{Supporting Information}

General. All the reactions were carried out under air atmosphere. Dioxane was dried by sodium and freshly distilled. Anhydrous $\mathrm{Cu}(\mathrm{OTf})_{2}$ and $\mathrm{AgF}$ were purchased from Alfa Aesar and Acros Chemical and used without further purification (Important! the sources of $\mathrm{Cu}(\mathrm{OTf})_{2}$ is very critical in this transformation.). $\mathrm{Pd}(\mathrm{OAc})_{2}$ was purchased from Alfa Aesar Chemical. ${ }^{1} \mathrm{H}$ NMR (300 MHz) and ${ }^{13} \mathrm{C}$ NMR $(75 \mathrm{MHz})$ were registered on Varian $300 \mathrm{M}$ spectrometers with $\mathrm{CDCl}_{3}$ as solvent and tetramethylsilane (TMS) as internal standard. Chemical shifts were reported in units (ppm) by assigning TMS resonance in the ${ }^{1} \mathrm{H}$ spectrum as $0.00 \mathrm{ppm}$ and $\mathrm{CDCl}_{3}$ resonance in the ${ }^{13} \mathrm{C}$ spectrum as $77.0 \mathrm{ppm}$. All coupling constants ( $J$ values) were reported in Hertz $(\mathrm{Hz})$. Column chromatography was performed on silica gel 200-300 mesh. IR, GC, MS, and HRMS were performed by the State-authorized Analytical Center in Peking University.

\section{General procedures for ortho arylation of acetanilide:}

In a $30 \mathrm{~mL}$ Schlenck tube, Acetanilide 1a $\left(0.2 \mathrm{mmol}, 1.0\right.$ equiv), $\mathrm{Pd}(\mathrm{OAc})_{2}(0.01 \mathrm{mmol}$, 0.05 equiv), anhydrous $\mathrm{Cu}(\mathrm{OTf})_{2}(0.4 \mathrm{mmol}, 2.0$ equiv) and anhydrous $\mathrm{AgF}(0.4 \mathrm{mmol}$, 2.0 equiv) was dissolved in $5.0 \mathrm{~mL}$ of dried 1, 4-Dioxane, then (trimethoxyl)phenysilane ( $80 \mu \mathrm{L}, 0.4 \mathrm{mmol}, 2.0$ equiv) was added via syringe. The tube was sealed with a Teflon lined cap, and the reaction mixture was stirred and heated to $110^{\circ} \mathrm{C}$ in an oil bath for $48 \mathrm{~h}$. After the reaction was completed, the dark solid was removed by filtration through Celite, and the Celite bed was washed with $3 \times 5 \mathrm{~mL}$ diethyl ether. In most cases, the combined filtrate was immediately concentrated, and the residue was purified by silica gel chromatography with a proper eluent. The purified material was dried under oil-pump vacuum. 
$\mathrm{N}$-Biphenyl-2-yl-acetamide (3aa). ${ }^{1}$ Following the general procedures, the reaction was performed on a $0.2 \mathrm{mmol}$ scale at $110{ }^{\circ} \mathrm{C}$ for $36 \mathrm{~h}$. A white powder was obtained after silica gel chromatography (70:30 hexanes:EtOAc), yield $0.031 \mathrm{~g}(74 \%)$ (With $\mathrm{Cu}(\mathrm{OTf})_{2}$ from different companies, such as Aldrich and Acros, the reaction could take place. However, the starting material 1a could not completely convert into product 3aa occasionally and the yield was ranged from $45-70 \%$ with the recovered starting material 1a). ${ }^{1} \mathrm{H}$ NMR $\left(300 \mathrm{MHz}, \mathrm{CDCl}_{3}\right) \delta 8.24(\mathrm{~d}, J=7.80 \mathrm{~Hz}, 1 \mathrm{H}), 7.51-7.33(\mathrm{~m}, 6 \mathrm{H})$, 7.25-7.15 (m, 3H), 2.01 (s, 3H); ${ }^{13} \mathrm{C}$ NMR (75 MHz, $\left.\mathrm{CDCl}_{3}\right) \delta 168.19,138.11,134.62$, $132.19,130.01,129.16,129.02,128.35,127.90,124.32,121.68,24.52 ; \mathrm{m} / \mathrm{z}(\mathrm{EI}) 211\left(\mathrm{M}^{+}\right.$, 33.3\%), 169 (100\%); IR (KBr plate, $\mathrm{CDCl}_{3}$ ) v 3261, 3031, 1663, 1520, 1445, 1293, 757.

$N$-(4'-Methoxy-biphenyl-2-yl)-acetamide (3ac). ${ }^{2}$ Following the general procedures, the reaction was performed on a $0.2 \mathrm{mmol}$ and $10 \mathrm{~mol} \% \mathrm{Pd}(\mathrm{OAc})_{2}$ scale at $110^{\circ} \mathrm{C}$ for $48 \mathrm{~h}$. A white powder was obtained after silica gel chromatography (50:50 hexanes:EtOAc), yield $0.034 \mathrm{~g}(71 \%)$. ${ }^{1} \mathrm{H}$ NMR $\left(300 \mathrm{MHz}, \mathrm{CDCl}_{3}\right) \delta 8.22(\mathrm{~d}, J=8.10 \mathrm{~Hz}, 1 \mathrm{H}), 7.51(\mathrm{~d}, J$ $=7.80 \mathrm{~Hz}, 1 \mathrm{H}), 7.36-7.18(\mathrm{~m}, 6 \mathrm{H}), 7.01(\mathrm{~d}, J=9.00 \mathrm{~Hz}, 2 \mathrm{H}), 3.86(\mathrm{~s}, 3 \mathrm{H}), 2.03(\mathrm{~s}, 3 \mathrm{H})$; ${ }^{13} \mathrm{C}$ NMR $\left(75 \mathrm{MHz}, \mathrm{CDCl}_{3}\right) \delta 168.25,159.23,134.71,131.94,130.28,130.07,128.79$, $127.98,124.31,123.97,121.60,119.75,114.38,55.25,24.43 ; \mathrm{m} / \mathrm{z}$ (EI) $241\left(\mathrm{M}^{+}, 58.8 \%\right)$, 199 (100\%); IR (KBr plate, $\left.\mathrm{CDCl}_{3}\right)$ v 3267, 1663, 1517, 1445, 1298, 1246, 1179, 1035, 834,760 .

$N$-(4'-Methyl-biphenyl-2-yl)-acetamide (3ad). ${ }^{2}$ Following the general procedures, the reaction was performed on a $0.2 \mathrm{mmol}$ scale at $110^{\circ} \mathrm{C}$ for $48 \mathrm{~h}$. A white powder was obtained after silica gel chromatography (65:35 hexanes:EtOAc), yield $0.028 \mathrm{~g}(62 \%) .{ }^{1} \mathrm{H}$ NMR (300 MHz, $\left.\mathrm{CDCl}_{3}\right) \delta 8.25(\mathrm{~d}, J=7.80 \mathrm{~Hz}, 1 \mathrm{H}), 7.37-7.17(\mathrm{~m}, 8 \mathrm{H}), 2.42(\mathrm{~s}, 3 \mathrm{H})$, $2.03(\mathrm{~s}, 3 \mathrm{H}) ;{ }^{13} \mathrm{C}$ NMR $\left(75 \mathrm{MHz}, \mathrm{CDCl}_{3}\right) \delta 168.21,137.75,135.01,134.67,132.01$, 130.04, 129.75, 129.03, 128.17, 124.24, 121.41, 24.61, 21.18; $\mathrm{m} / \mathrm{z}$ (EI) $225\left(\mathrm{M}^{+}, 46.9 \%\right)$, $183(100 \%)$; IR ( $\mathrm{KBr}$ plate, $\left.\mathrm{CDCl}_{3}\right)$ v 3264, 3024, 1663, 1520, 1445, 1303, 1289, 821, 757.

$N$-(4'-Fluoro-biphenyl-2-yl)-acetamide (3ae). ${ }^{2}$ Following the general procedures, the reaction was performed on a $0.2 \mathrm{mmol}$ scale at $110^{\circ} \mathrm{C}$ for $48 \mathrm{~h}$. A white powder was obtained after silica gel chromatography (65:35 hexanes:EtOAc), yield $0.029 \mathrm{~g}(63 \%) .{ }^{1} \mathrm{H}$ NMR (300 MHz, $\left.\mathrm{CDCl}_{3}\right) \delta 8.20(\mathrm{~d}, J=8.40 \mathrm{~Hz}, 1 \mathrm{H}), 7.40-7.32(\mathrm{~m}, 3 \mathrm{H}), 7.27-7.15(\mathrm{~m}$, $4 \mathrm{H}), 7.06(\mathrm{~s}, 1 \mathrm{H}), 2.03(\mathrm{~s}, 3 \mathrm{H}) ;{ }^{13} \mathrm{C} \mathrm{NMR}\left(75 \mathrm{MHz}, \mathrm{CDCl}_{3}\right) \delta$ 168.29, 164.06, 160.77, 134.60, 134.05, 131.47, 130.96, 130.86, 130.09, 128.54, 124.55, 122.09, 116.16, 115.88, 115.75, 24.50; m/z (EI) $229\left(\mathrm{M}^{+}, 98.2 \%\right), 185(100 \%)$; IR ( $\mathrm{KBr}$ plate, $\left.\mathrm{CDCl}_{3}\right) \vee 3259$, $3039,1663,1515,1446,1222,1159,838,759$.

$N$-(4'-Chloro-biphenyl-2-yl)-acetamide (3af). Following the general procedures, the reaction was performed on a $0.2 \mathrm{mmol}$ scale at $110^{\circ} \mathrm{C}$ for $48 \mathrm{~h}$. A white powder was obtained after silica gel chromatography (65:35 hexanes:EtOAc), yield $0.024 \mathrm{~g}(46 \%) .{ }^{1} \mathrm{H}$ $\operatorname{NMR}\left(300 \mathrm{MHz}, \mathrm{CDCl}_{3}\right) \delta 8.16(8.16, J=8.10 \mathrm{~Hz}, 1 \mathrm{H}), 7.47-7.27(\mathrm{~m}, 5 \mathrm{H}), 7.20(\mathrm{~d}, J=$ $6.60 \mathrm{~Hz}, 2 \mathrm{H}), 7.09(\mathrm{~s}, 1 \mathrm{H}), 2.03(\mathrm{~s}, 3 \mathrm{H}) ;{ }^{13} \mathrm{C}$ NMR $\left(75 \mathrm{MHz}, \mathrm{CDCl}_{3}\right) \delta 168.35,136.56$, $134.44,133.99,131.42,130.52,129.95,129.19,128.68,124.69,122.36,24.44 ; \mathrm{m} / \mathrm{z}$ (EI) $247\left(\mathrm{M}^{+},{ }^{37} \mathrm{Cl}, 12.5 \%\right), 245\left(\mathrm{M}^{+},{ }^{35} \mathrm{Cl}, 34.1 \%\right), 203(100 \%) ; \mathrm{IR}\left(\mathrm{KBr}\right.$ plate, $\left.\mathrm{CDCl}_{3}\right) \vee 3248$, 
3027, 1652, 1524, 1291, 857, 701. Anal. Calcd. for $\mathrm{C}_{14} \mathrm{H}_{12} \mathrm{NOCl}$ : C, 68.44; H, 4.92; N, 5.70. Found: C, 68.29; H, 4.99; N, 5.59.

$N$-(3'-Methoxy-biphenyl-2-yl)-acetamide (3ag). ${ }^{2}$ Following the general procedures, the reaction was performed on a $0.2 \mathrm{mmol}$ scale at $110{ }^{\circ} \mathrm{C}$ for $48 \mathrm{~h}$. A white powder was obtained after silica gel chromatography (50:50 hexanes:EtOAc), yield $0.025 \mathrm{~g}(52 \%) .{ }^{1} \mathrm{H}$ NMR $\left(300 \mathrm{MHz}, \mathrm{CDCl}_{3}\right) \delta 8.28(\mathrm{~d}, J=8.10 \mathrm{~Hz}, 1 \mathrm{H}), 7.39(\mathrm{~m}, 2 \mathrm{H}), 7.34-7.17(\mathrm{~m}, 3 \mathrm{H})$, 6.97-6.89 (m, 3H), $3.84(\mathrm{~s}, 3 \mathrm{H}), 2.03(\mathrm{~s}, 3 \mathrm{H}) ;{ }^{13} \mathrm{C}$ NMR $\left(75 \mathrm{MHz}, \mathrm{CDCl}_{3}\right) \delta 168.24$, 160.02 , 139.43, 134.62, 131.83, 130.08, 129.87, 128.43, 124.20, 121.38, 121.30, 114.71, 113.54, 55.29, 24.65; m/z (EI) $241\left(\mathrm{M}^{+}, 52.7 \%\right), 199(100 \%)$; IR (KBr plate, $\left.\mathrm{CDCl}_{3}\right) v$ $3267,1664,1586,1522,1442,1303,759$.

$N$-Biphenyl-2-yl-benzamide (3ca). Following the general procedures, the reaction was performed on a $0.2 \mathrm{mmol}$ scale at $110^{\circ} \mathrm{C}$ for $48 \mathrm{~h}$. A white powder was obtained after silica gel chromatography (90:10 hexanes:EtOAc), yield $0.021 \mathrm{~g} \mathrm{(38 \% ).}{ }^{1} \mathrm{H}$ NMR (300 $\left.\mathrm{MHz} \mathrm{CDCl}_{3}\right) \delta 8.53(\mathrm{~d}, J=8.40 \mathrm{~Hz}, 1 \mathrm{H}), 8.01(\mathrm{~s}, 1 \mathrm{H}), 7.60(\mathrm{~d}, J=7.20 \mathrm{~Hz}, 2 \mathrm{H}), 7.54$ -7.29 (m, 8H), 7.24-7.19 (m, 3H); ${ }^{13} \mathrm{C}$ NMR (75 MHz, $\left.\mathrm{CDCl}_{3}\right) \delta$ 164.97, 138.02, 134.87, $134.73,132.33,131.69,129.96,129.51,129.32,129.19,128.98,128.71,128.57,128.15$, 126.77, 124.35, 121.13, 115.33; m/z (EI) $273\left(\mathrm{M}^{+}, 55.4 \%\right), 105$ (100\%); IR (KBr plate, $\left.\mathrm{CDCl}_{3}\right) \vee 3295,1664,1581,1519,1489,1449,1306,1073,1027,796,759,702$; Anal. Calcd. For $\mathrm{C}_{19} \mathrm{H}_{15} \mathrm{NO}$ : C, 83.49; H, 5.53; N, 5.12. Found: C, 83.27; H, 5.63; N, 4.95.

$N$-Biphenyl-2-yl-3-phenyl-propionamide (3ha). Following the general procedures, the reaction was performed on a $0.2 \mathrm{mmol}$ scale at $110^{\circ} \mathrm{C}$ for $48 \mathrm{~h}$. A white powder was obtained after silica gel chromatography (90:10 hexanes:EtOAc), yield $0.028 \mathrm{~g}(46 \%) .{ }^{1} \mathrm{H}$ NMR $\left(300 \mathrm{MHz}, \mathrm{CDCl}_{3}\right) \delta 8.29(\mathrm{~d}, J=8.10 \mathrm{~Hz}, 1 \mathrm{H}), 7.43-7.29(\mathrm{~m}, 5 \mathrm{H}), 7.27-7.14(\mathrm{~m}$, $8 \mathrm{H}), 7.07(\mathrm{~s}, 1 \mathrm{H}), 2.95(\mathrm{~d}, J=7.05 \mathrm{~Hz}, 2 \mathrm{H}), 2.47(\mathrm{~d}, J=7.65 \mathrm{~Hz}, 2 \mathrm{H}) ;{ }^{13} \mathrm{C}$ NMR $(75$ $\left.\mathrm{MHz}_{\mathrm{CDCl}}\right) \delta 170.11,140.50,137.95,134.50,132.05,129.98,129.83,129.16,129.02$, $128.77,128.54,128.36,128.30,128.14,127.87,127.24,126.28,124.27,121.48,39.54$, 31.35; m/z (EI) $301\left(\mathrm{M}^{+}, 98.8 \%\right), 167(100 \%)$; IR (KBr plate, $\left.\mathrm{CDCl}_{3}\right)$ v 3281, 3025, 1662, 1584, 1518, 1494, 1478, 1447, 1278, 1218, 1075, 751, 699; Anal. Calcd. For $\mathrm{C}_{21} \mathrm{H}_{19} \mathrm{NO}$ : C, 83.69; H, 6.35; N, 4.65. Found: C, 83.54; H, 6.46; N, 4.81.

$N$-(5-Methoxy-biphenyl-2-yl)-acetamide (3ja). Following the general procedures, the reaction was performed on a $0.2 \mathrm{mmol}$ scale at $110^{\circ} \mathrm{C}$ for $48 \mathrm{~h}$. A white powder was obtained after silica gel chromatography (70:30 hexanes:EtOAc), yield $0.033 \mathrm{~g}(68 \%) .{ }^{1} \mathrm{H}$ NMR $\left(300 \mathrm{MHz}, \mathrm{CDCl}_{3}\right) \delta 7.98(\mathrm{~d}, J=5.40 \mathrm{~Hz}, 1 \mathrm{H}), 7.49-7.35(\mathrm{~m}, 5 \mathrm{H}), 6.97-6.88(\mathrm{~m}$, $2 \mathrm{H}), 6.80(\mathrm{~d}, J=2.40 \mathrm{~Hz}, 1 \mathrm{H}), 3.81(\mathrm{~s}, 3 \mathrm{H}), 1.99(\mathrm{~s}, 3 \mathrm{H}) ;{ }^{13} \mathrm{C} \mathrm{NMR}\left(75 \mathrm{MHz}, \mathrm{CDCl}_{3}\right) \delta$ $168.41,156.41,138.19,134.70,129.11,129.02$, 128.89, 127.92, 127.61, 124.33, 115.35, 113.36, 55.46, 24.19; m/z (EI) $241\left(\mathrm{M}^{+}, 58.8 \%\right), 199$ (100\%); IR (KBr plate, $\left.\mathrm{CDCl}_{3}\right) v$ $3285,1669,1522,1442,1315,1172,1126,1115,1035,758$; Anal. Calcd. For $\mathrm{C}_{15} \mathrm{H}_{15} \mathrm{NO}_{2}$ : C, 74.67; H, 6.27; N, 5.81. Found: C, 74.54; H, 6.20; N, 5.68.

Acetic acid 6-acetylamino-biphenyl-3-yl ester (3ka). Following the general procedures, the reaction was performed on a $0.2 \mathrm{mmol}$ scale at $110^{\circ} \mathrm{C}$ for $48 \mathrm{~h}$. A white powder was obtained after silica gel chromatography (90:10 hexanes:EtOAc), yield $0.034 \mathrm{~g}(64 \%) .{ }^{1} \mathrm{H}$ 
NMR $\left(300 \mathrm{MHz}, \mathrm{CDCl}_{3}\right) \delta 8.29(\mathrm{~d}, J=8.70 \mathrm{~Hz}, 1 \mathrm{H}), 7.50-7.43(\mathrm{~m}, 3 \mathrm{H}), 7.38(\mathrm{~d}, J=8.10$ $\mathrm{Hz}, 2 \mathrm{H}), 7.12-7.01(\mathrm{~m}, 2 \mathrm{H}), 7.00(\mathrm{~s}, 1 \mathrm{H}), 2.30(\mathrm{~s}, 3 \mathrm{H}), 2.03(\mathrm{~s}, 3 \mathrm{H}) ;{ }^{13} \mathrm{C}$ NMR $(75 \mathrm{MHz}$, $\left.\mathrm{CDCl}_{3}\right) \delta 168.24,165.17,149.26,137.18,133.62,133.40,132.38,130.11,129.31,129.11$, 128.54, 128.27, 123.11, 122.80, 17.86, 16.52; m/z (EI) $269\left(\mathrm{M}^{+}, 50.6 \%\right)$, 167 (100\%); HRMS: Anal. Calcd. for $\mathrm{C}_{16} \mathrm{H}_{15} \mathrm{NO}_{3}$ 269.10519. Found: 269.10537; IR (KBr plate, $\mathrm{CDCl}_{3}$ ) $v 3280,1736,1669,1518,1451,1247,800,754,703$.

Benzoic acid 6-acetylamino-biphenyl-3-yl ester (3la). Following the general procedures, the reaction was performed on a $0.2 \mathrm{mmol} \mathrm{scale}$ at $110{ }^{\circ} \mathrm{C}$ for $48 \mathrm{~h}$. A white powder was obtained after silica gel chromatography (90:10 hexanes:EtOAc), yield 0.042 $\mathrm{g}(64 \%) .{ }^{1} \mathrm{H}$ NMR $\left(300 \mathrm{MHz}, \mathrm{CDCl}_{3}\right) \delta 8.32(\mathrm{~d}, J=8.40 \mathrm{~Hz}, 1 \mathrm{H}), 7.19(\mathrm{~d}, J=10.20 \mathrm{~Hz}$, 2H), $7.66-7.53(\mathrm{~m}, 1 \mathrm{H}), 7.52-7.38(\mathrm{~m}, 6 \mathrm{H}), 7.23-7.13(\mathrm{~m}, 3 \mathrm{H}), 2.03(\mathrm{~s}, 3 \mathrm{H}) ;{ }^{13} \mathrm{C} \mathrm{NMR}$ $\left(75 \mathrm{MHz}, \mathrm{CDCl}_{3}\right) \delta 168.24,165.17,146.96,137.18,133.62,133.40,132.38,130.11$, 129.31, 129.11, 128.54, 128.27, 123.11, 122.80, 121.36, 24.52; m/z (EI) $331\left(\mathrm{M}^{+}, 48.3 \%\right)$, 105 (100\%); HRMS: Anal. Calcd. for $\mathrm{C}_{21} \mathrm{H}_{17} \mathrm{NO}_{3}$ 331.12084. Found: 331.12101; IR (KBr plate, $\left.\mathrm{CDCl}_{3}\right) \vee 3280,1736,1669,1518,1451,1247,800,754,703$.

$N$-(5-Methyl-biphenyl-2-yl)-acetamide (3ma). ${ }^{2}$ Following the general procedures, the reaction was performed on a $0.2 \mathrm{mmol}$ scale at $110{ }^{\circ} \mathrm{C}$ for $48 \mathrm{~h}$. A white powder was obtained after silica gel chromatography (70:30 hexanes:EtOAc), yield $0.032 \mathrm{~g}(71 \%) .{ }^{1} \mathrm{H}$ NMR $\left(300 \mathrm{MHz}, \mathrm{CDCl}_{3}\right) \delta 8.04(\mathrm{~d}, J=8.10 \mathrm{~Hz}, 1 \mathrm{H}), 7.49-7.33(\mathrm{~m}, 5 \mathrm{H}), 7.18-7.11(\mathrm{~m}$, 2H), $7.06(\mathrm{~s}, 1 \mathrm{H}), 2.34(\mathrm{~s}, 3 \mathrm{H}), 1.99(\mathrm{~s}, 3 \mathrm{H}) ;{ }^{13} \mathrm{C} \mathrm{NMR}\left(75 \mathrm{MHz}, \mathrm{CDCl}_{3}\right) \delta 168.26$, 138.24, 134.07, 132.50, 131.93, 130.56, 129.08, 128.87, 128.81, 127.73, 122.09, 24.36, 20.79; m/z (EI) $225\left(\mathrm{M}^{+}, 40.0 \%\right), 183(100 \%)$; IR (KBr plate, $\left.\mathrm{CDCl}_{3}\right)$ v 3281, 3023, 1655, $1525,1443,1298,1133,830,754$.

$N$-(5-Chloro-biphenyl-2-yl)-acetamide (3na). ${ }^{2}$ Following the general procedures, the reaction was performed on a $0.2 \mathrm{mmol}$ scale at $110^{\circ} \mathrm{C}$ for $48 \mathrm{~h}$. A white powder was obtained after silica gel chromatography (70:30 hexanes:EtOAc), yield $0.030 \mathrm{~g}(61 \%) .{ }^{1} \mathrm{H}$ NMR $\left(300 \mathrm{MHz}, \mathrm{CDCl}_{3}\right) \delta 8.24(\mathrm{~d}, J=8.70 \mathrm{~Hz}, 1 \mathrm{H}), 7.51-7.45(\mathrm{~m}, 3 \mathrm{H}), 7.37-7.31(\mathrm{~m}$, $3 \mathrm{H}), 7.25(\mathrm{~d}, J=8.70 \mathrm{~Hz}, 1 \mathrm{H}), 7.23(\mathrm{~s}, 1 \mathrm{H}), 2.02(\mathrm{~s}, 3 \mathrm{H}) ;{ }^{13} \mathrm{C} \mathrm{NMR}\left(75 \mathrm{MHz}, \mathrm{CDCl}_{3}\right) \delta$ $168.24,136.75,133.58,133.25,129.75,129.49,129.24,128.99,128.46,128.22,122.78$, 114.44, 24.55; m/z (EI) $247\left(\mathrm{M}^{+},{ }^{37} \mathrm{Cl}\right), 245\left(\mathrm{M}^{+},{ }^{35} \mathrm{Cl}\right)$; IR $\left(\mathrm{KBr}\right.$ plate, $\left.\mathrm{CDCl}_{3}\right) \vee 3266$, 2924, 1666, 1510, 1480, 1442, 1294, 1097, 787, 755.

$N$-(5-Acetyl-biphenyl-2-yl)-acetamide(3oa). Following the general procedures, the reaction was performed on a $0.2 \mathrm{mmol}$ scale at $110^{\circ} \mathrm{C}$ for $48 \mathrm{~h}$. A white powder was obtained purified by silica gel chromatography (hexanes:EtOAc, 65:35), yield $0.030 \mathrm{~g}$ (59\%). ${ }^{1} \mathrm{H}$ NMR $\left(300 \mathrm{MHz}, \mathrm{CDCl}_{3}\right) \delta 8.51(\mathrm{~d}, J=8.40 \mathrm{~Hz}, 1 \mathrm{H}), 7.96(\mathrm{q}, J=4.20 \mathrm{~Hz}$, $1 \mathrm{H}), 7.85(\mathrm{~d}, J=5.40 \mathrm{~Hz}, 1 \mathrm{H}), 7.53-7.51(\mathrm{~m}, 3 \mathrm{H}), 7.49-7.37(\mathrm{~m}, 3 \mathrm{H}), 2.59(\mathrm{~s}, 3 \mathrm{H}), 2.04$ $(\mathrm{s}, 3 \mathrm{H}) ;{ }^{13} \mathrm{C} \mathrm{NMR}\left(75 \mathrm{MHz}, \mathrm{CDCl}_{3}\right) \delta 197.03,168.38,139.10,136.94,132.52,130.24$, 129.40, 129.15, 128.55, 128.42, 119.98, 26.48, 24.86; m/z (EI) $253\left(\mathrm{M}^{+}, 50.6 \%\right) 196$ (100\%); HRMS: Anal. Calcd. for $\mathrm{C}_{16} \mathrm{H}_{15} \mathrm{NO}_{2}$ 283.11028. Found: 253.11064; IR (KBr plate, $\left.\mathrm{CDCl}_{3}\right) \vee 3311,2983,1712,1514,1443,1296,1235,1108,753,702$.

$N$-(4-Methoxy-biphenyl-2-yl)-acetamide (3pa). Following the general procedures, the 
reaction was performed on a $0.2 \mathrm{mmol}$ scale at $110{ }^{\circ} \mathrm{C}$ for $48 \mathrm{~h}$. A white powder was S8obtained after silica gel chromatography (50:50 hexanes:EtOAc), yield 0.025 g (52\%). ${ }^{1} \mathrm{H}$ NMR $\left(300 \mathrm{MHz}, \mathrm{CDCl}_{3}\right) \delta 7.98(\mathrm{~d}, J=2.70 \mathrm{~Hz}, 1 \mathrm{H}), 7.49-7.33(\mathrm{~m}, 5 \mathrm{H}), 7.23(\mathrm{~s}$, $1 \mathrm{H}), 7.14(\mathrm{~d}, J=8.40 \mathrm{~Hz}, 1 \mathrm{H}), 6.74(\mathrm{~d}, J=2.70 \mathrm{~Hz}, 1 \mathrm{H}), 3.85(\mathrm{~s}, 3 \mathrm{H}), 2.02(\mathrm{~s}, 3 \mathrm{H}) ;{ }^{13} \mathrm{C}$ NMR $\left(75 \mathrm{MHz}, \mathrm{CDCl}_{3}\right) \delta 168.27,159.48,137.90,135.58,130.68,129.36,129.07$, 127.61, 124.26, 110.52, 106.21, 55.40, 24.74; m/z (EI) $241\left(\mathrm{M}^{+}, 52.7 \%\right), 199$ (100\%); IR $\left(\mathrm{KBr}\right.$ plate, $\left.\mathrm{CDCl}_{3}\right) \vee 3262,2934,1666,1583,1527,14666,1303,1236,1166,1049,764$, 701; Anal. Calcd. for $\mathrm{C}_{15} \mathrm{H}_{15} \mathrm{NO}_{2}$ : C, 74.67; H, 6.27; N, 5.81. Found: C, 74.57; H, 6.15; N, 5.68 .

$N$-(3-Methoxy-biphenyl-2-yl)-acetamide (3qa). ${ }^{3}$ Following the general procedures, the reaction was performed on a $0.2 \mathrm{mmol}$ scale at $110^{\circ} \mathrm{C}$ for $48 \mathrm{~h}$. A white powder was S8obtained after silica gel chromatography (50:50 hexanes:EtOAc), yield 0.039 g (80\%). ${ }^{1} \mathrm{H}$ NMR $\left(300 \mathrm{MHz}, \mathrm{CDCl}_{3}\right) \delta$ 7.36-7.31 (m, 6H), $6.93(\mathrm{~d}, J=8.10 \mathrm{~Hz}, 2 \mathrm{H}), 6.70(\mathrm{~s}, 1 \mathrm{H})$, $3.86(\mathrm{~s}, 3 \mathrm{H}), 1.99(\mathrm{~d}, J=5.10 \mathrm{~Hz}, 2 \mathrm{H}), 1.65(\mathrm{~s}, 1 \mathrm{H}) ;{ }^{13} \mathrm{C} \mathrm{NMR}\left(75 \mathrm{MHz}, \mathrm{CDCl}_{3}\right) \delta$ 169.62, 154.99, 140.98, 139.37, 128.94, 128.53, 128.34, 127.47, 122.20, 122.29, 110.43, 55.86, 23.10; m/z (EI) $241\left(\mathrm{M}^{+}, 52.7 \%\right), 199$ (100\%); IR (KBr plate, $\left.\mathrm{CDCl}_{3}\right) \vee 3128$, 2974, 1656, 1466, 1260, 1121, 1020, 759, 705.

$N$-(3, 5-Dimethyl-biphenyl-2-yl)-acetamide (3ra). ${ }^{2}$ Following the general procedures, the reaction was performed on a $0.2 \mathrm{mmol}$ scale at $110{ }^{\circ} \mathrm{C}$ for $48 \mathrm{~h}$. A white powder was obtained after silica gel chromatography (hexanes:EtOAc, 65:35), yield 0.035 g (73\%). ${ }^{1} \mathrm{H}$ NMR $\left(300 \mathrm{MHz}, \mathrm{CDCl}_{3}\right) \delta$ 7.43-7.30 (m, 6H), 7.09 (s, 1H), $7.00(\mathrm{~s}, 1 \mathrm{H}), 6.60(\mathrm{~s}, 1 \mathrm{H})$, 2.34 (s, 3H), 2.29 (s, 3H), $1.99(\mathrm{~s}, 3 \mathrm{H}) ;{ }^{13} \mathrm{C} \mathrm{NMR}\left(75 \mathrm{MHz}, \mathrm{CDCl}_{3}\right) \delta 169.58,139.68$, $139.37,137.21,136.52,130.84,129.87,128.98,128.80,128.58,128.46,128.25,23.03$, 20.96, 18.46; m/z (EI) $239\left(\mathrm{M}^{+}, 52.7 \%\right), 197$ (100\%); IR (KBr plate, $\left.\mathrm{CDCl}_{3}\right)$ v 3248, $3027,1652,1524,1291,857,701$.

$N$-(3-Methoxy-5-methyl-biphenyl-2-yl)-acetamide (3sa). Following the general procedures, the reaction was performed on a $0.2 \mathrm{mmol} \mathrm{scale}$ at $110{ }^{\circ} \mathrm{C}$ for $48 \mathrm{~h}$. A white powder was obtained after silica gel chromatography (65:35 hexanes:EtOAc), yield 0.033 $\mathrm{g}(65 \%) .{ }^{1} \mathrm{H}$ NMR $\left(300 \mathrm{MHz}, \mathrm{CDCl}_{3}\right) \delta 7.88(\mathrm{~s}, 1 \mathrm{H}), 7.45(\mathrm{~d}, J=6.90,2 \mathrm{H}), 7.39-7.32(\mathrm{~m}$, $3 \mathrm{H}), 7.18(\mathrm{~s}, 1 \mathrm{H}), 7.01(\mathrm{~s}, 1 \mathrm{H}), 3.88(\mathrm{~s}, 3 \mathrm{H}), 2.20(\mathrm{~s}, 3 \mathrm{H}), 2.02(\mathrm{~s}, 3 \mathrm{H}) ;{ }^{13} \mathrm{C} \mathrm{NMR}(75$ $\left.\mathrm{MHz}_{\mathrm{CDCl}}\right) \delta 168.28,157.31,138.17,133.25,131.63,129.53,128.97,127.43,123.83$, 122.56, 103.85, 55.49, 24.59, 15.70; m/z (EI) $255\left(\mathrm{M}^{+}, 52.7 \%\right), 213(100 \%)$; IR (KBr plate, $\left.\mathrm{CDCl}_{3}\right) \vee 3282,3003,1666,1517,1398,1233,1143,1050,753$, 702; Anal. Calcd. for $\mathrm{C}_{16} \mathrm{H}_{17} \mathrm{NO}_{2}$ : C, 75.27; H, 6.71; N, 5.49. Found: C, 75.33; H, 6.79; N, 5.53.

Acetic acid 6-acetylamino-4-methoxy-biphenyl-3-yl ester (3ta). Following the general procedures, the reaction was performed on a $0.2 \mathrm{mmol} \mathrm{scale}$ at $110{ }^{\circ} \mathrm{C}$ for $48 \mathrm{~h}$. A white powder was obtained after silica gel chromatography (50:50 hexanes:EtOAc), yield 0.037 g (62\%). ${ }^{1} \mathrm{H}$ NMR $\left(300 \mathrm{MHz}, \mathrm{CDCl}_{3}\right) \delta 8.30(\mathrm{~s}, 1 \mathrm{H}), 7.76(\mathrm{~s}, 1 \mathrm{H}), 7.53-7.34(\mathrm{~m}, 6 \mathrm{H})$, $3.98(\mathrm{~s}, 3 \mathrm{H}), 3.86(\mathrm{~s}, 3 \mathrm{H}), 2.06(\mathrm{~s}, 3 \mathrm{H}) ;{ }^{13} \mathrm{C} \mathrm{NMR}\left(75 \mathrm{MHz}, \mathrm{CDCl}_{3}\right) \delta 168.51,165.87$, $159.83,139.61,136.66,133.58,129.35,129.33,128.15,122.71,114.31,103.56,56.20$, 51.88, 25.02; m/z (EI) $299\left(\mathrm{M}^{+}, 52.7 \%\right), 226$ (100\%); IR (KBr plate, $\left.\mathrm{CDCl}_{3}\right) \vee 3422$, $3017,1719,1693,1580,1464,1399,1224,1090,1081$, 753; Anal. Calcd. for $\mathrm{C}_{17} \mathrm{H}_{14} \mathrm{NO}_{4}$ : 
C, 68.21; H, 5.72; N, 4.68; Found: C, 68.00; H, 5.80; N, 4.54.

\section{References:}

1. Chauhan, K. K.; Frost, C. G.; Love, I.; Waite, D. Synlett 1999, 1743-1744.

2. Tsang, W. C. P.; Zheng, N.; Buchwald, S. L. J. Am. Chem. Soc. 2005, 127, 14560-14561.

3. Kim, H. J.; Asif, R.; Chung, D. S.; Hong, J. I. Tetrahedron Lett. 2003, 44, 4355-4359. 


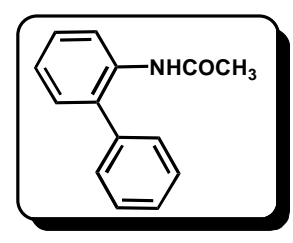

3aa
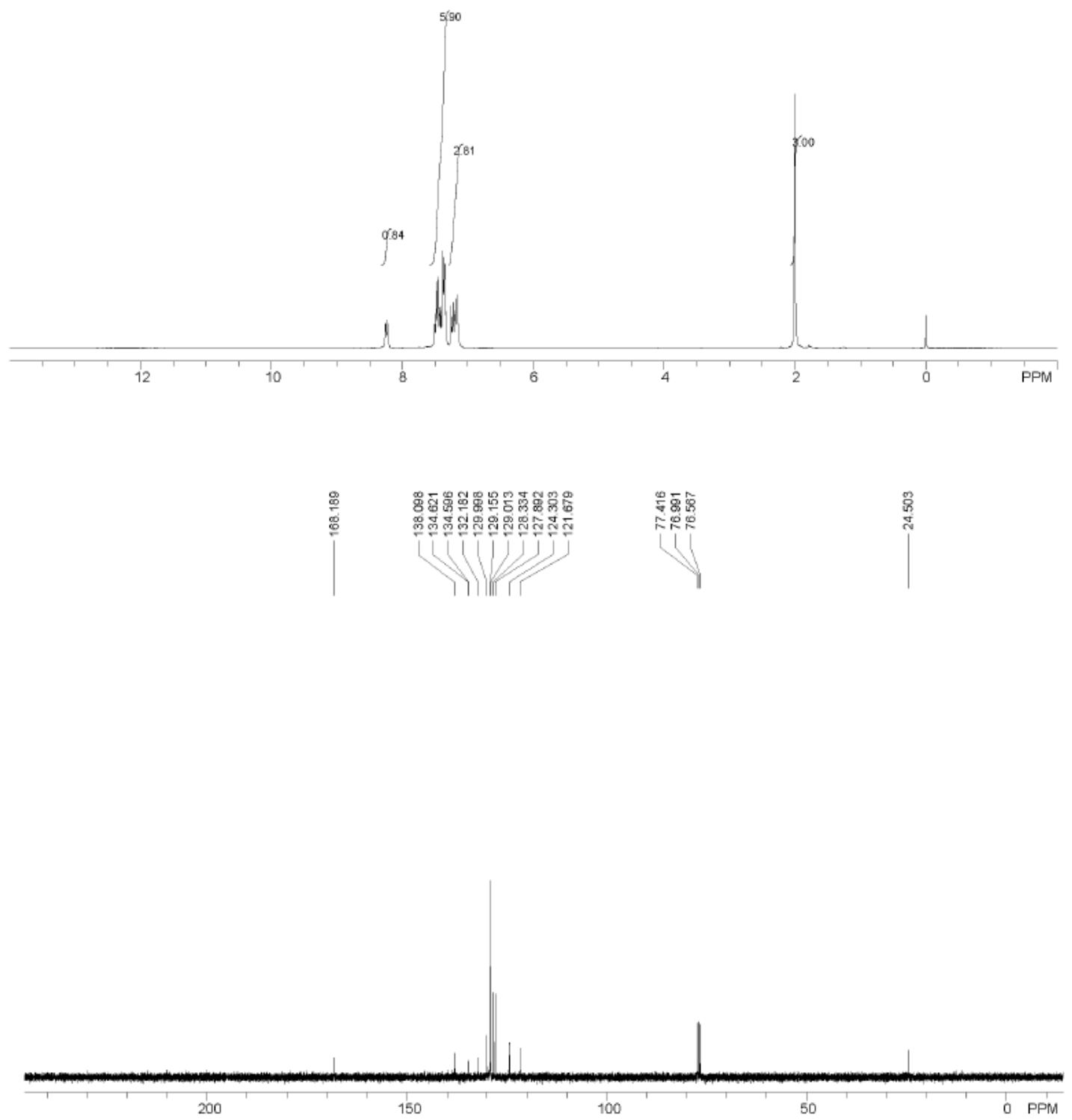


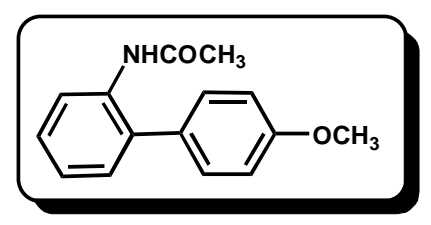

Зас
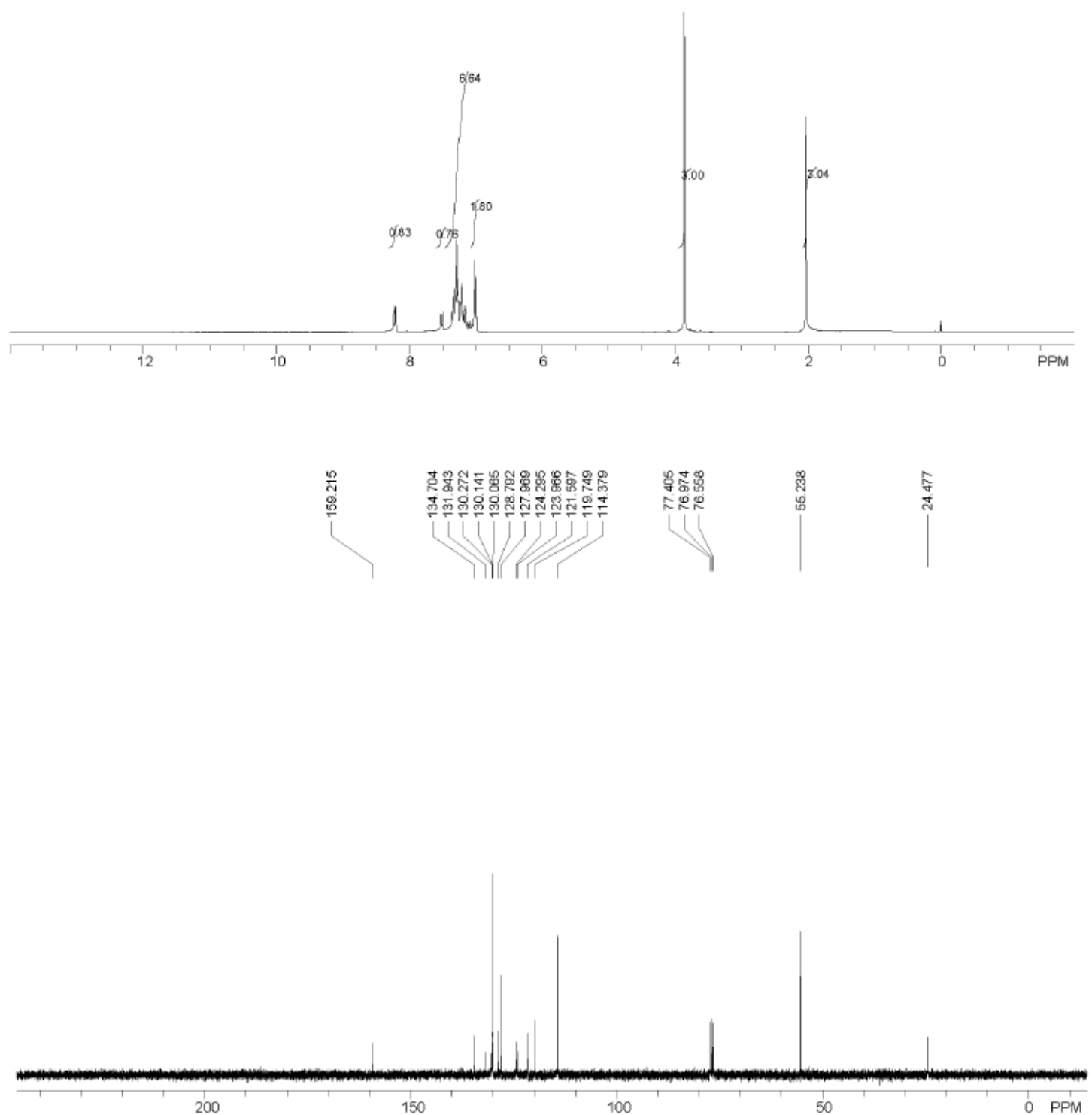


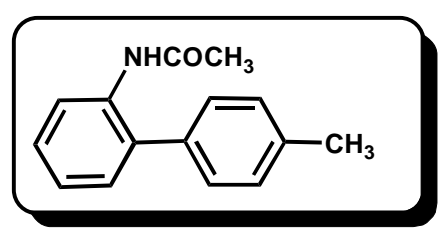

3ad
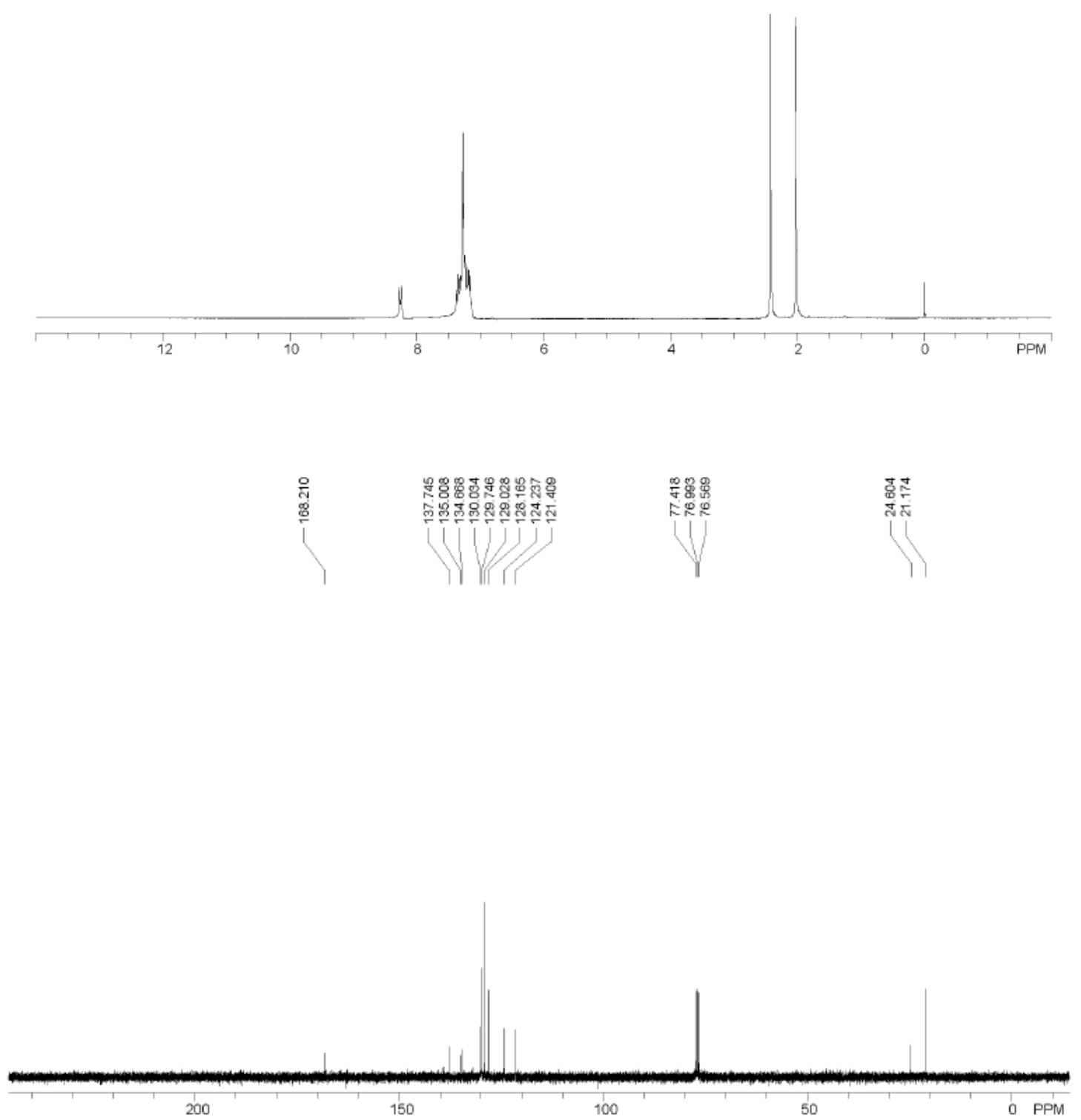


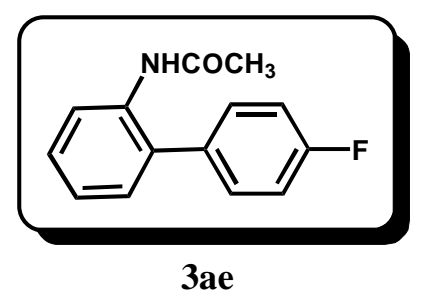

3ae
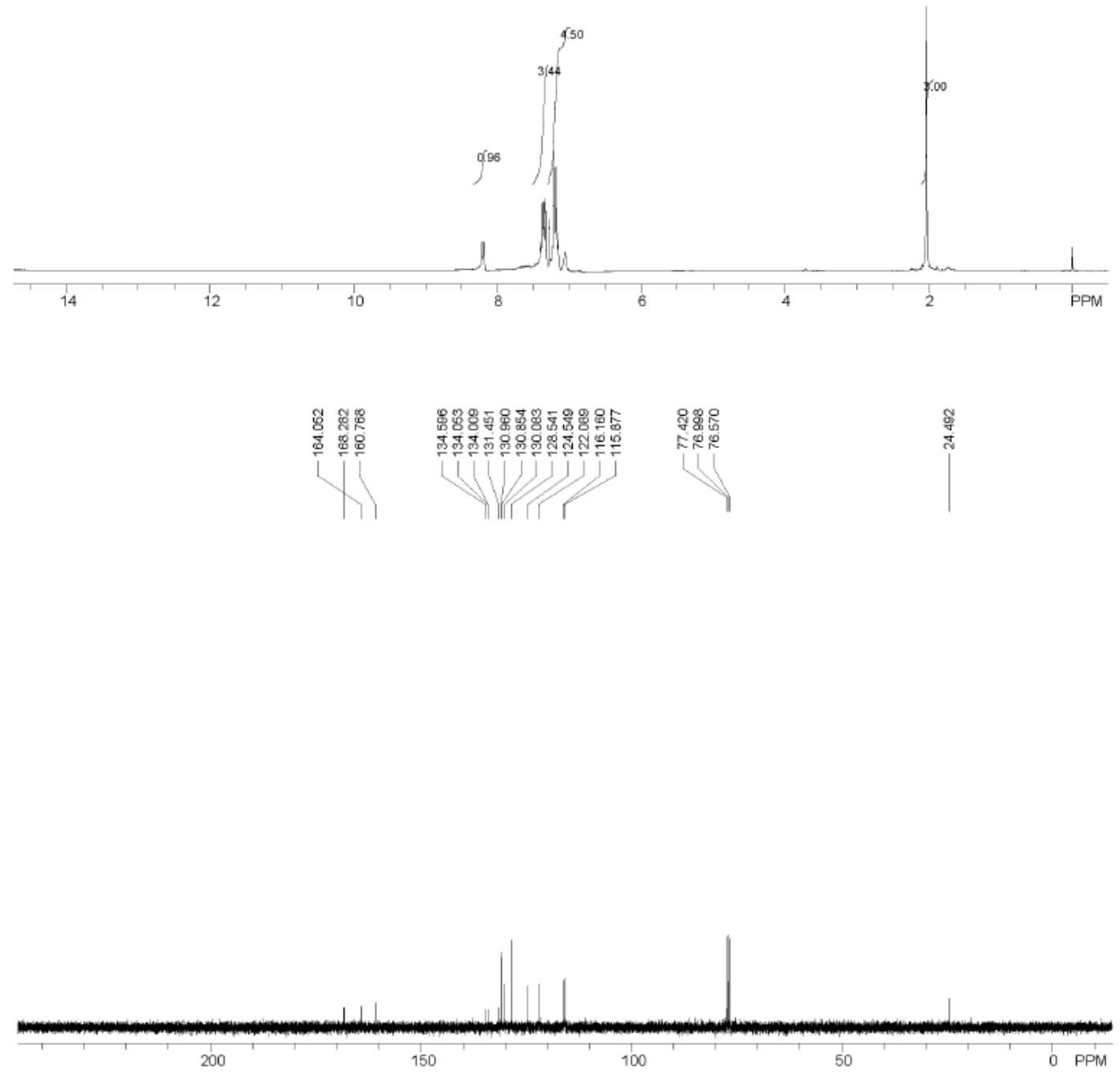

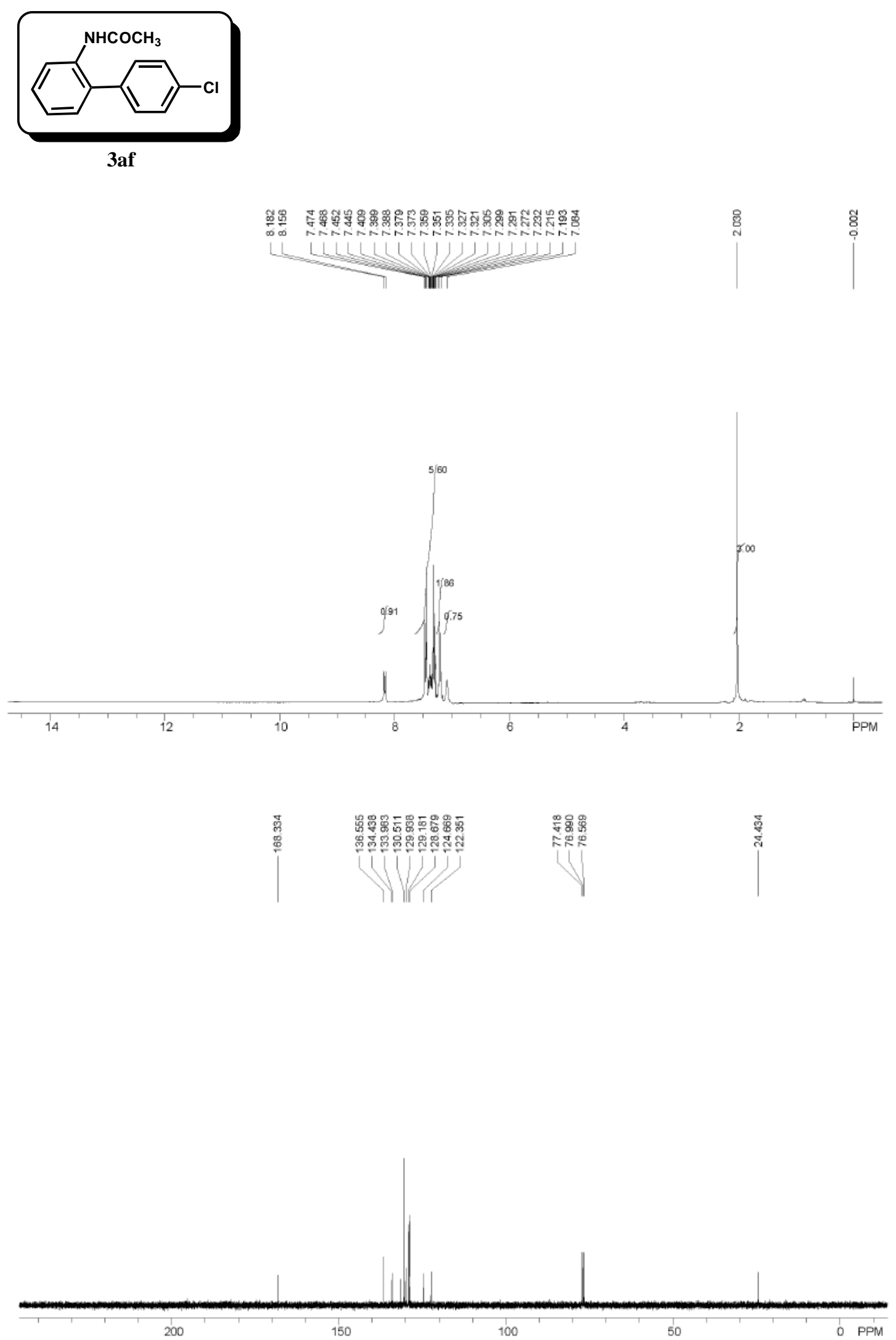


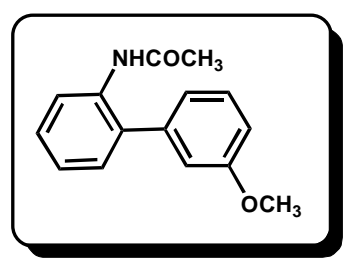

3ag
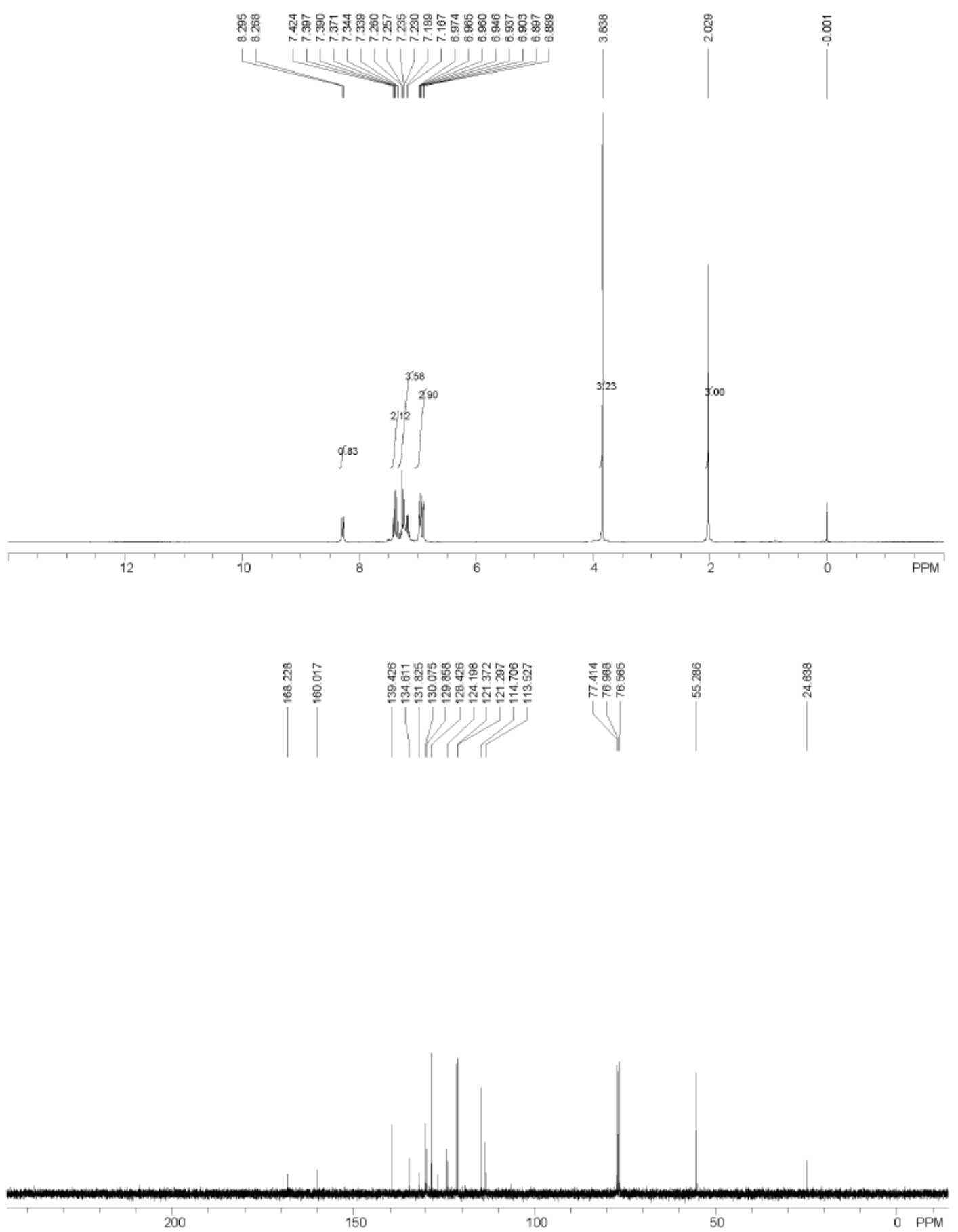

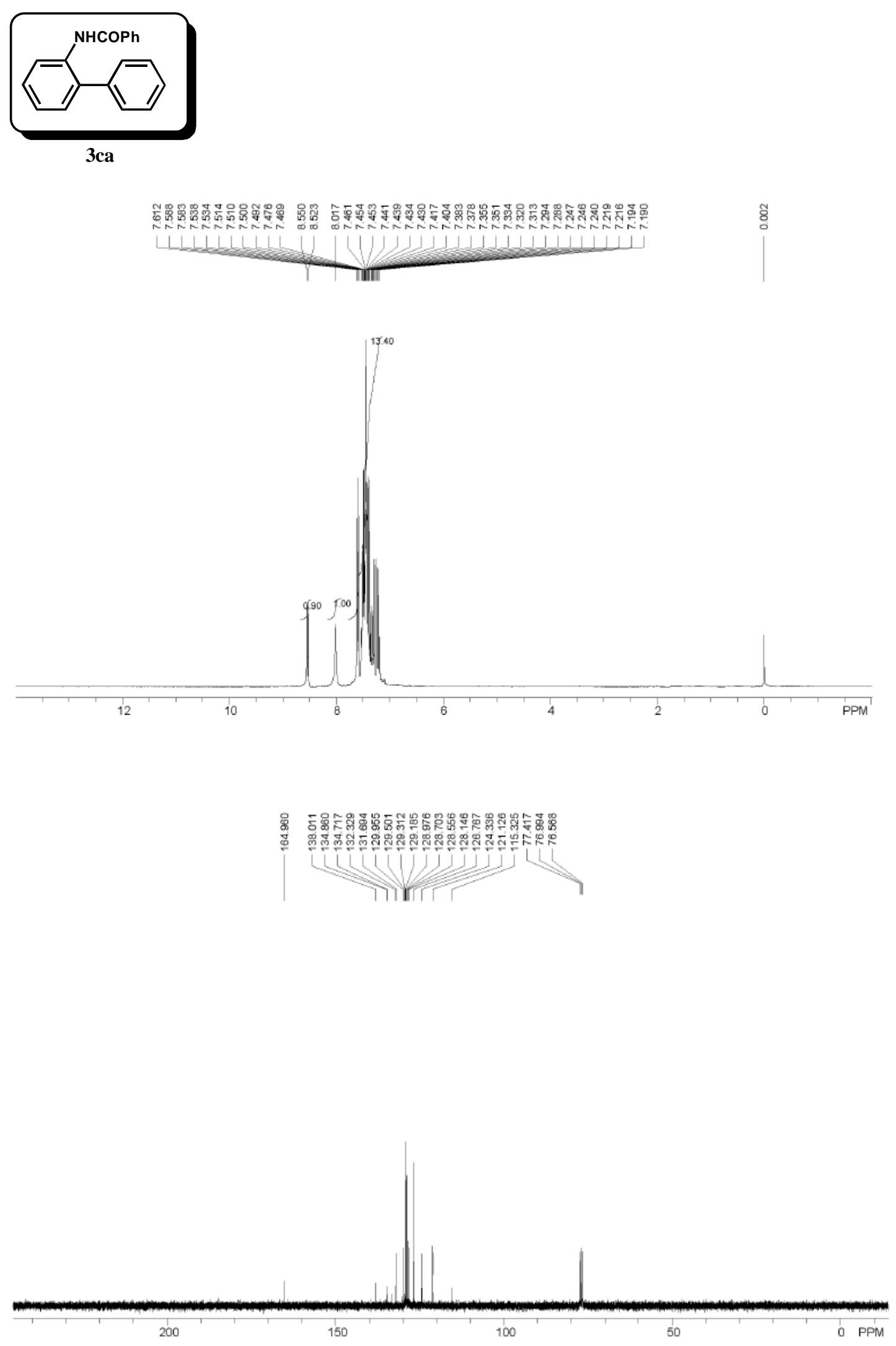


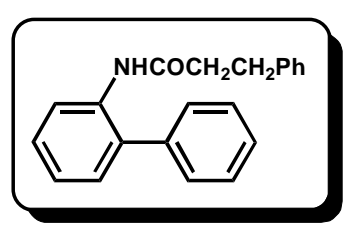

3ha
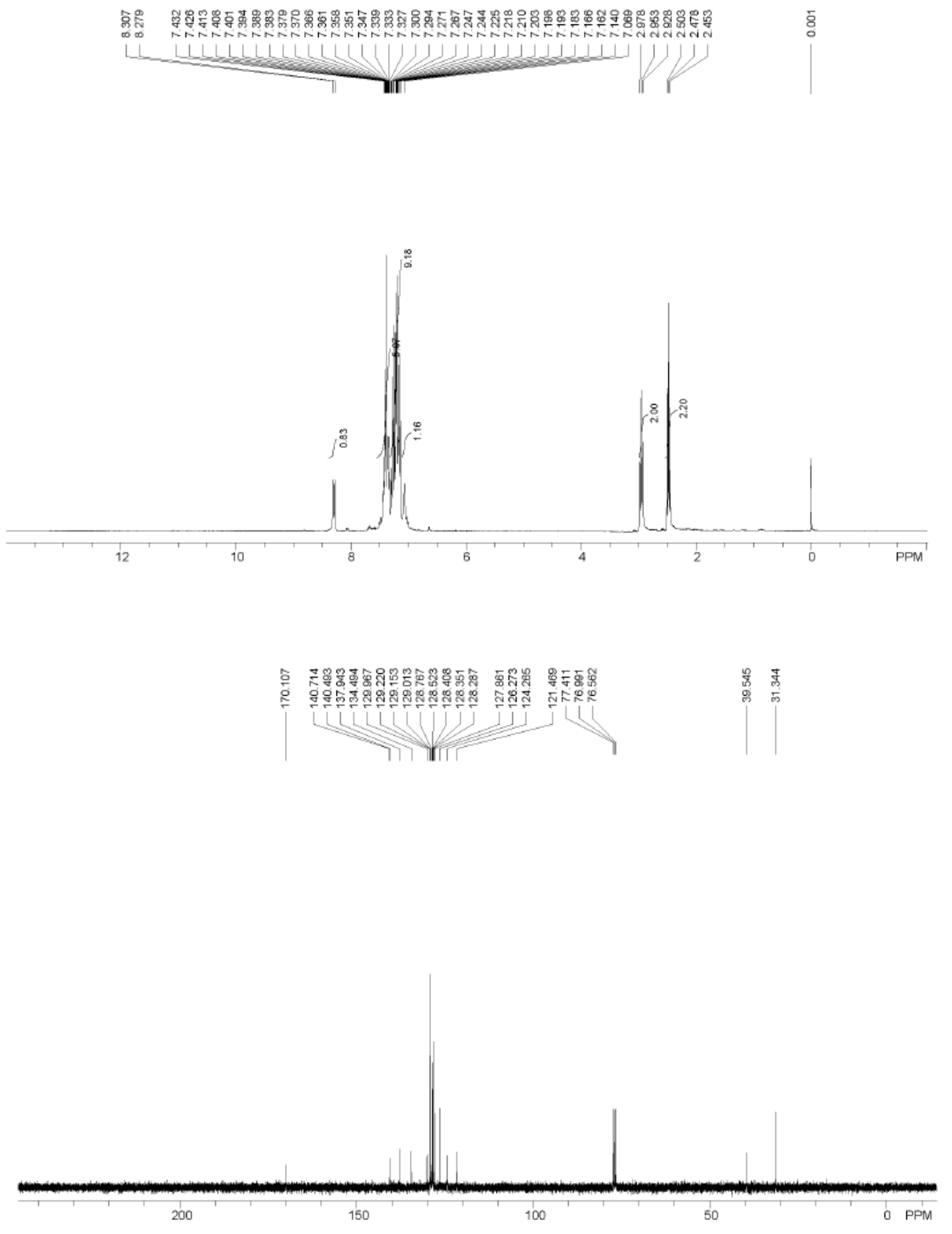


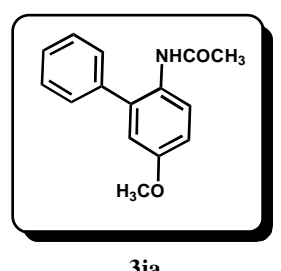

3ja
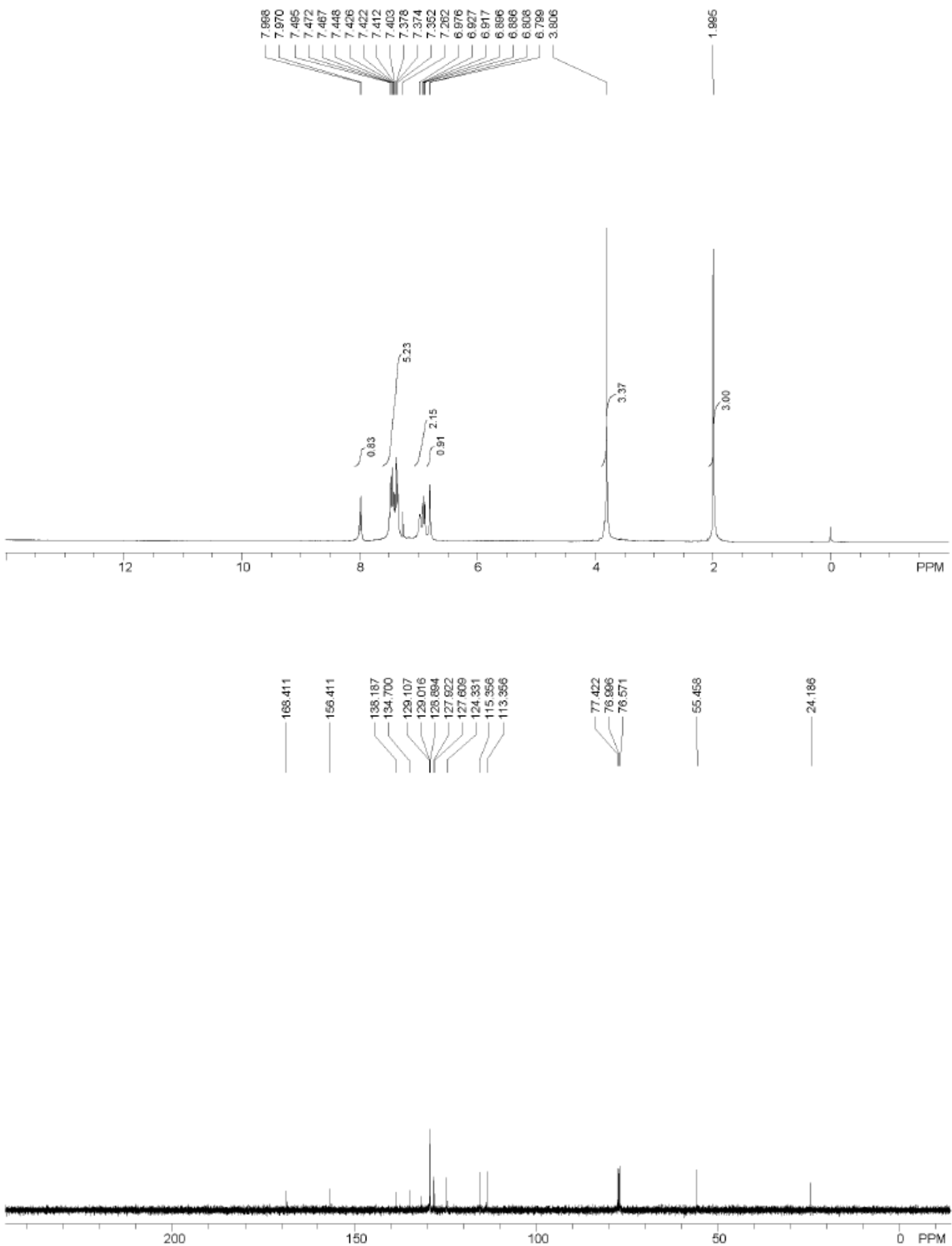

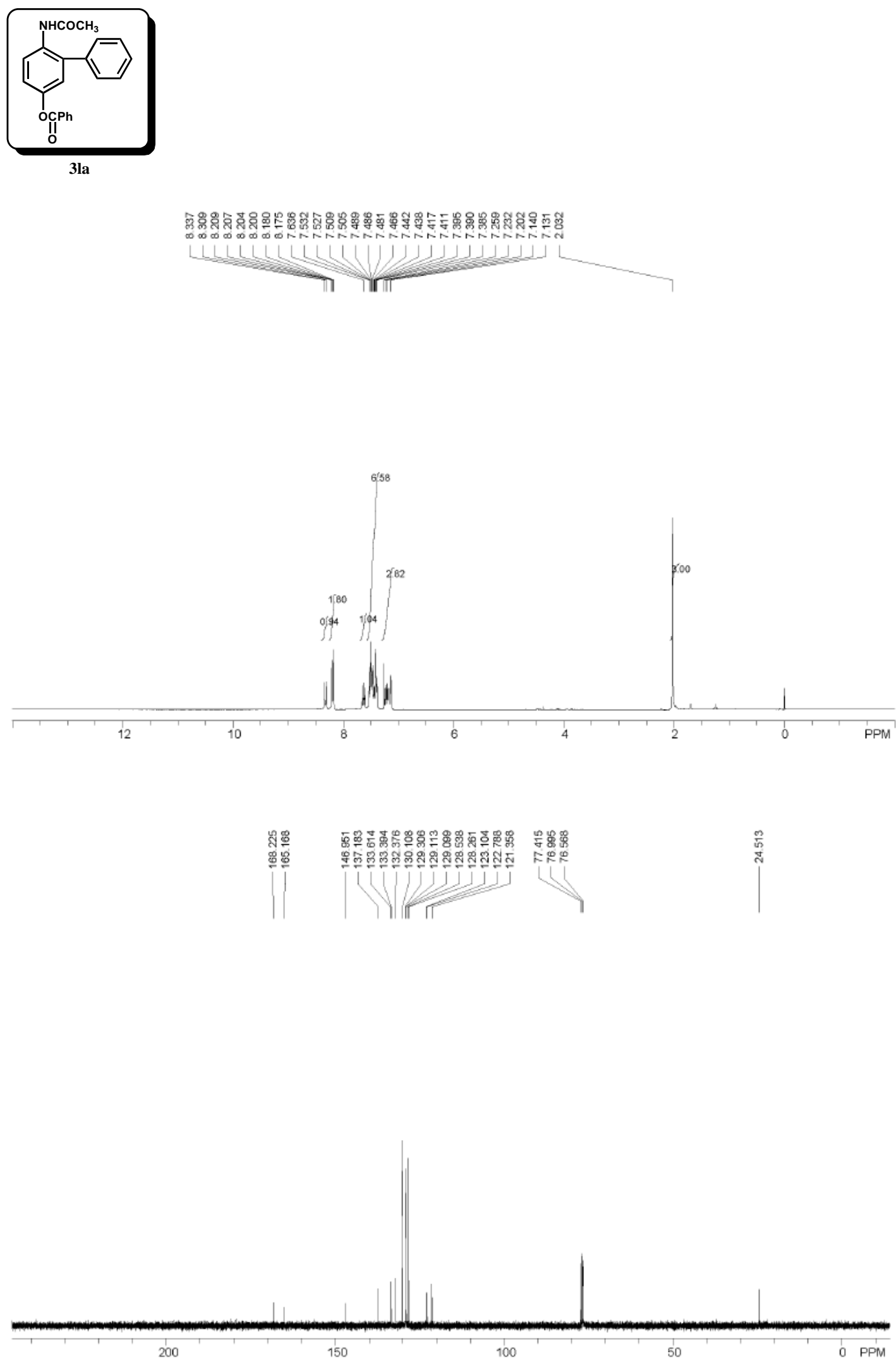

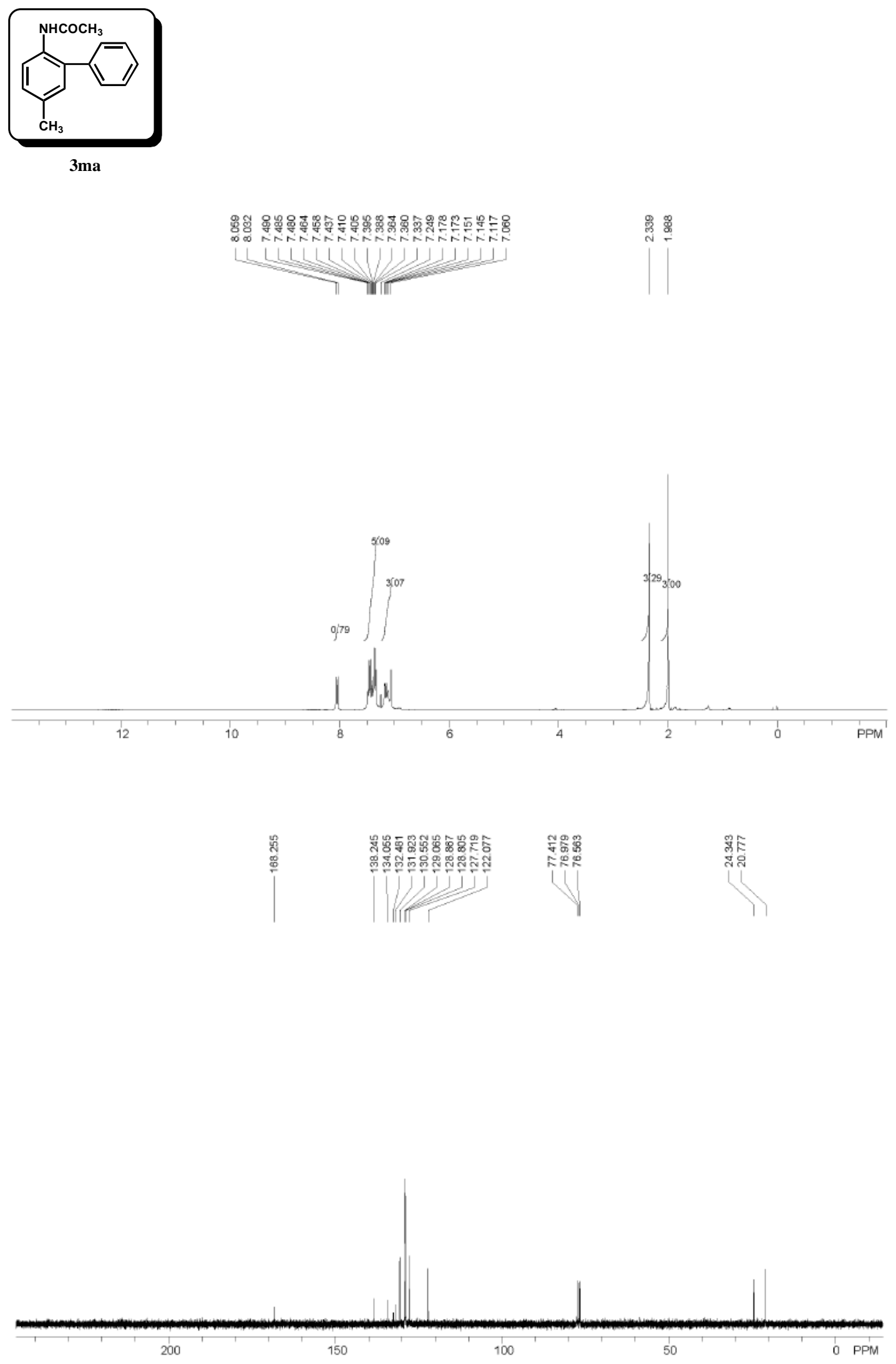


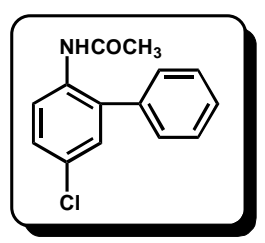

3na
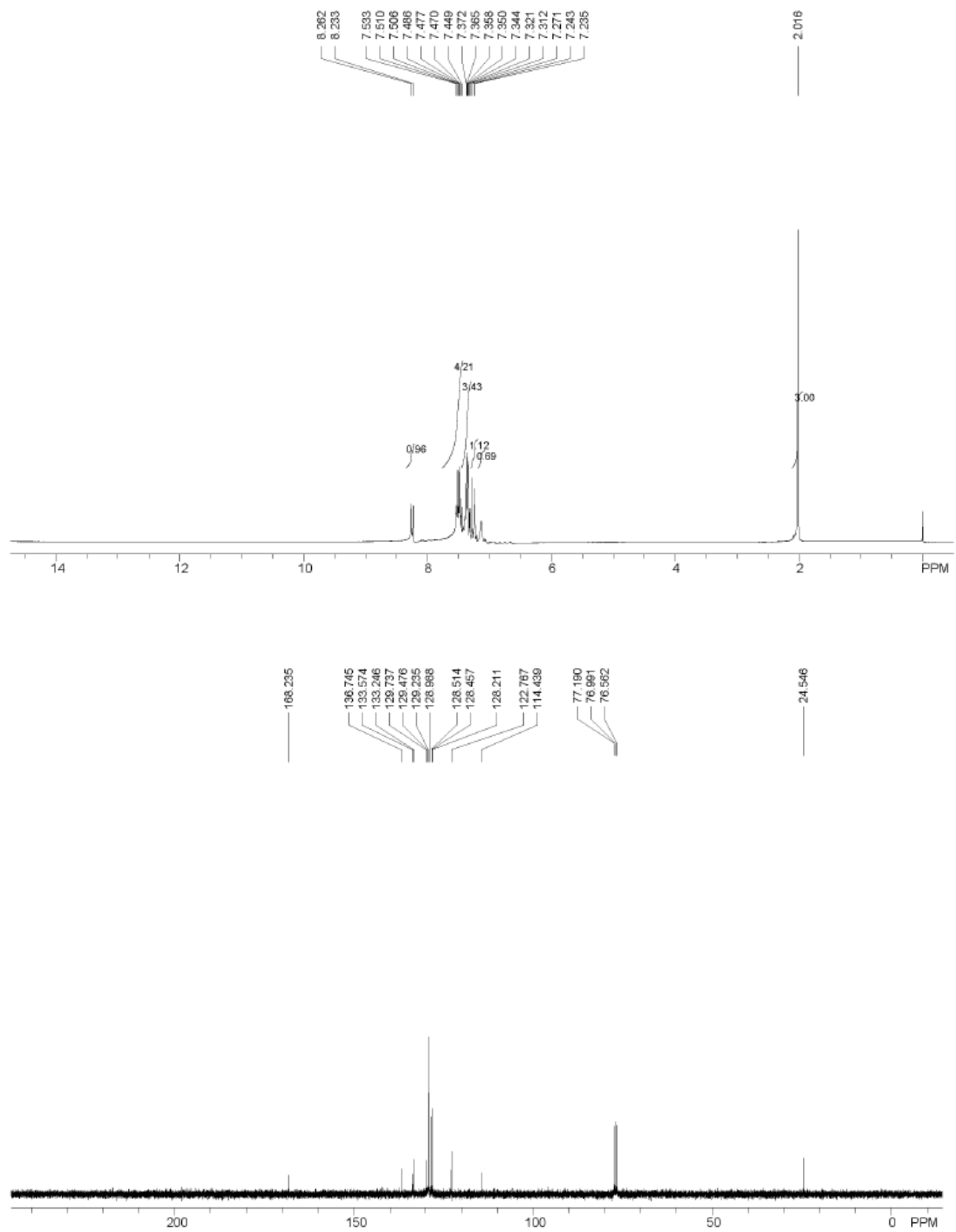

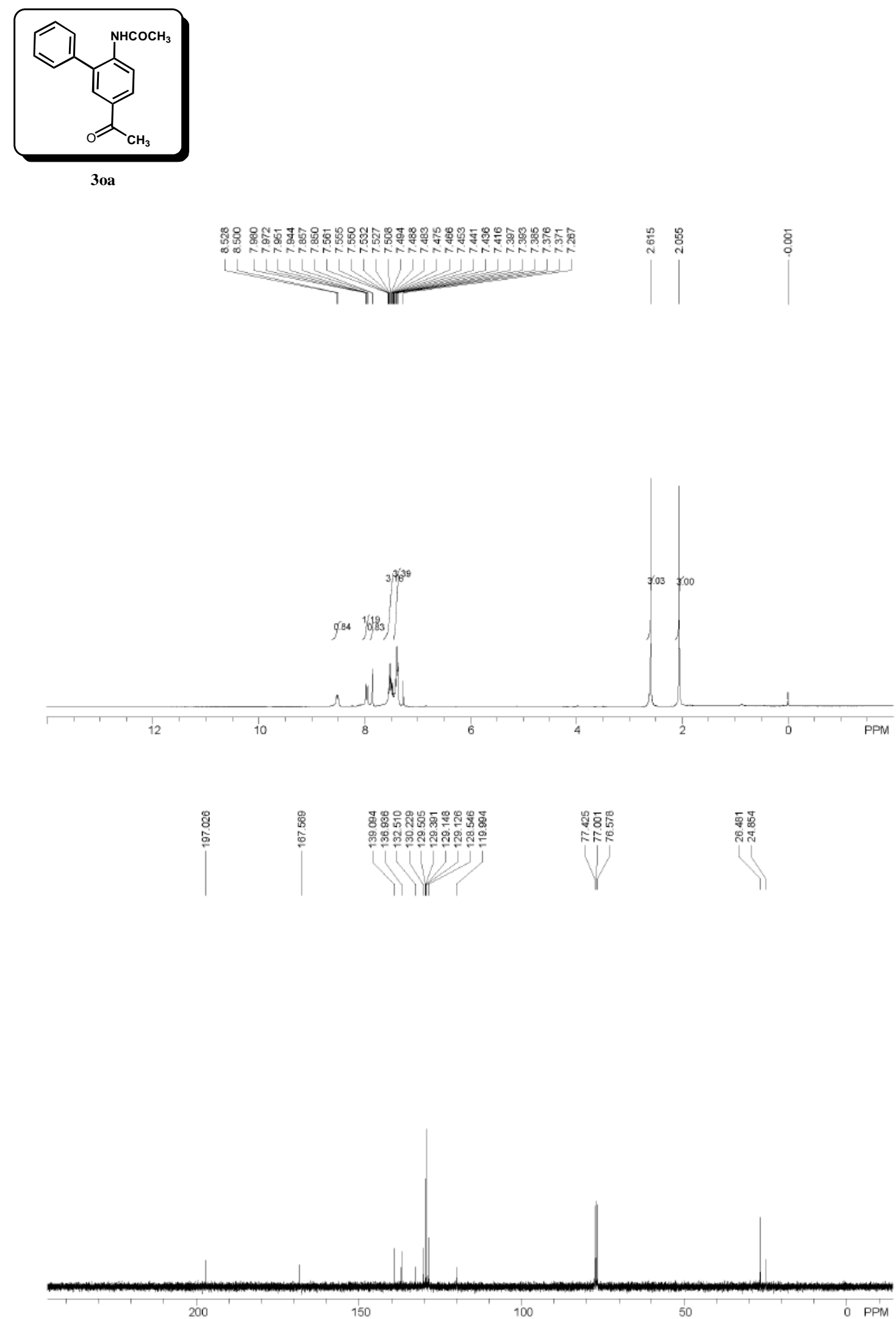


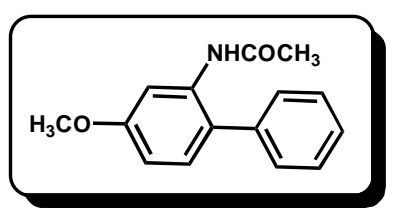

Зра

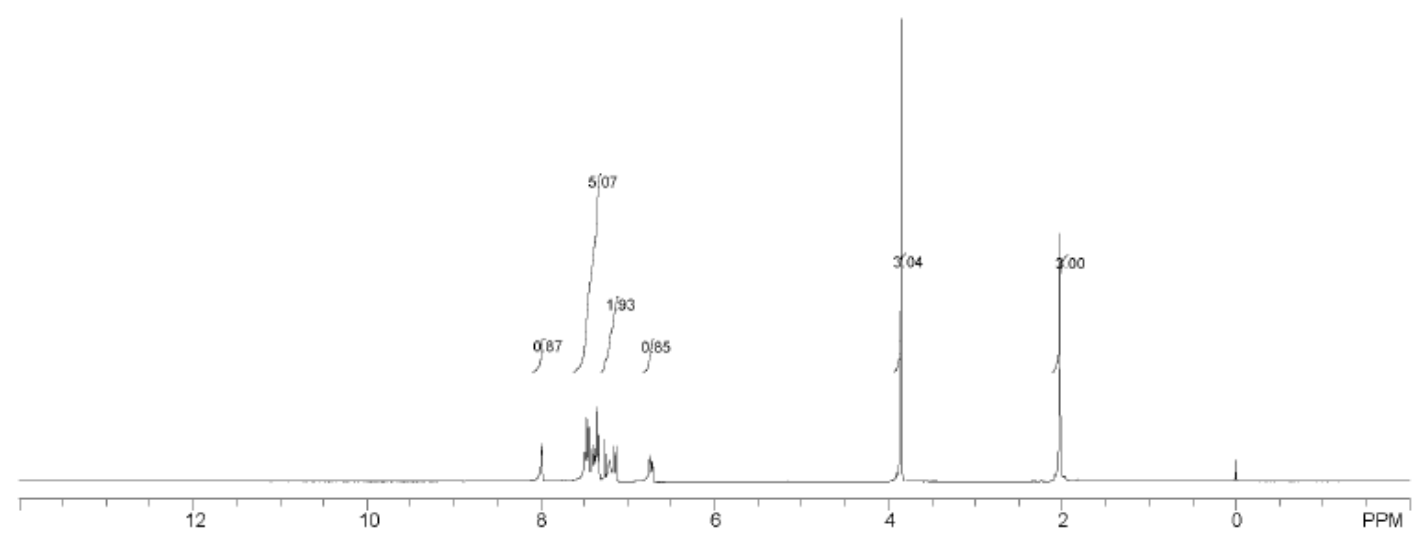

$\mid$

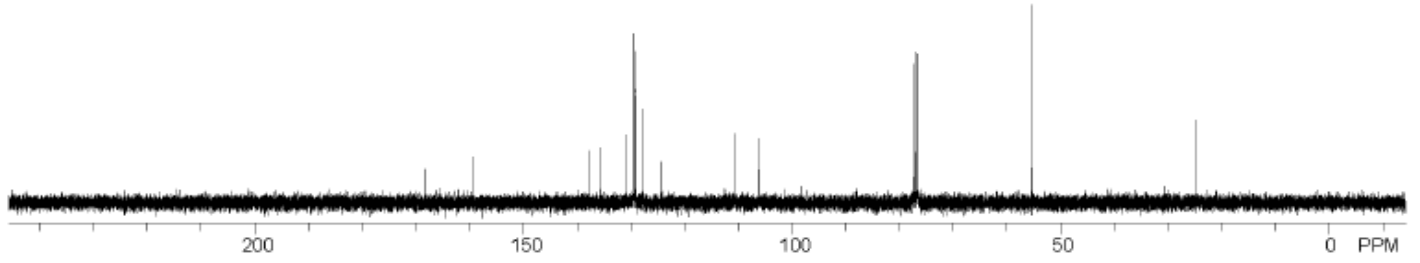




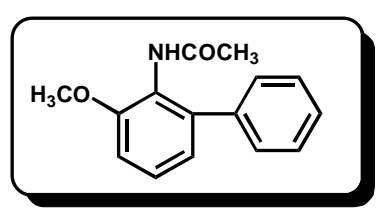

$3 q a$
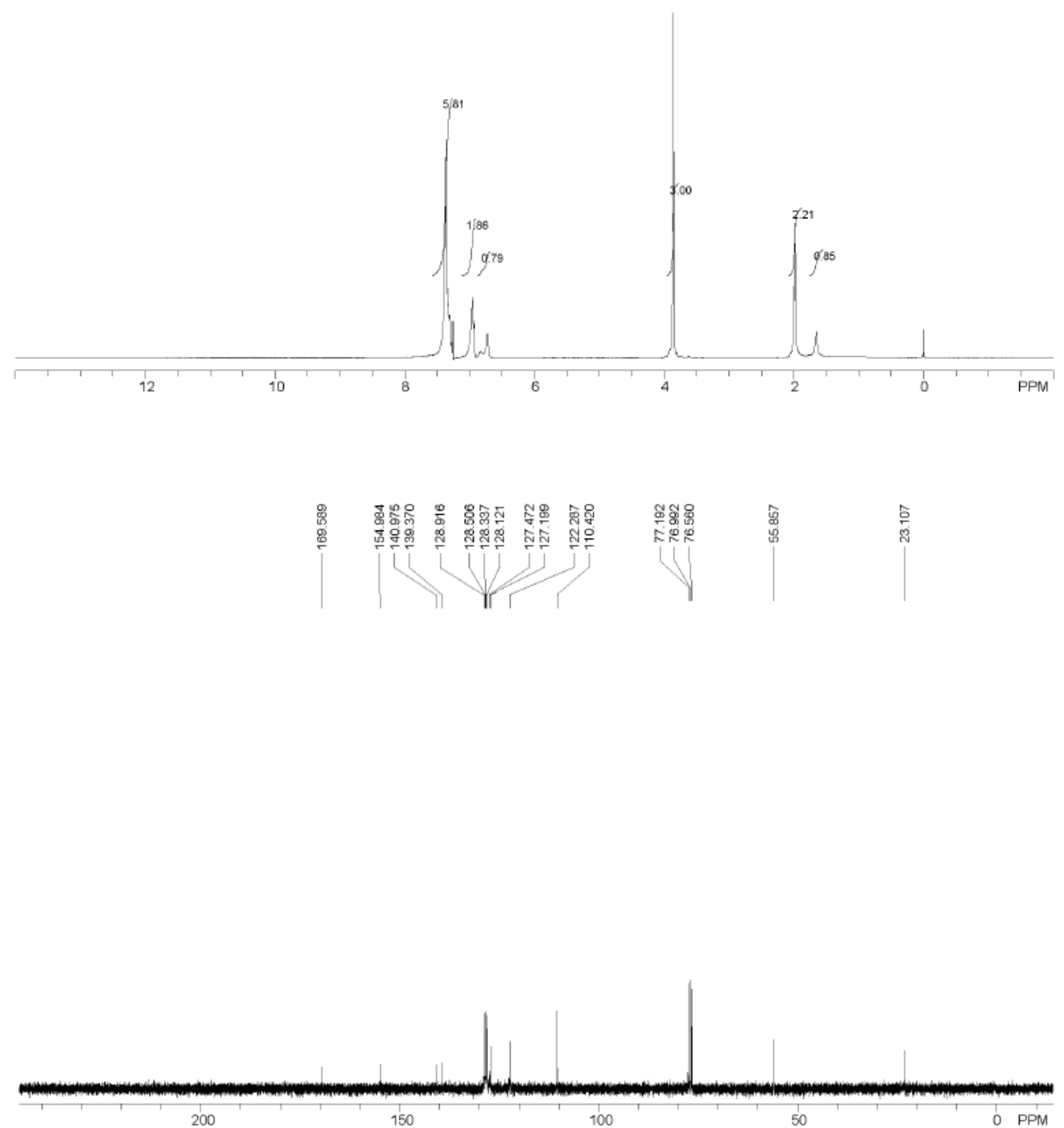


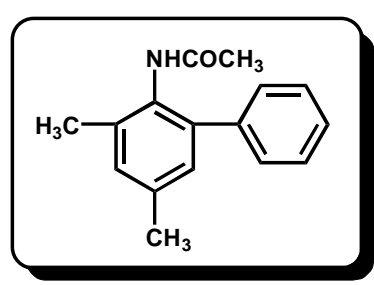

3ra
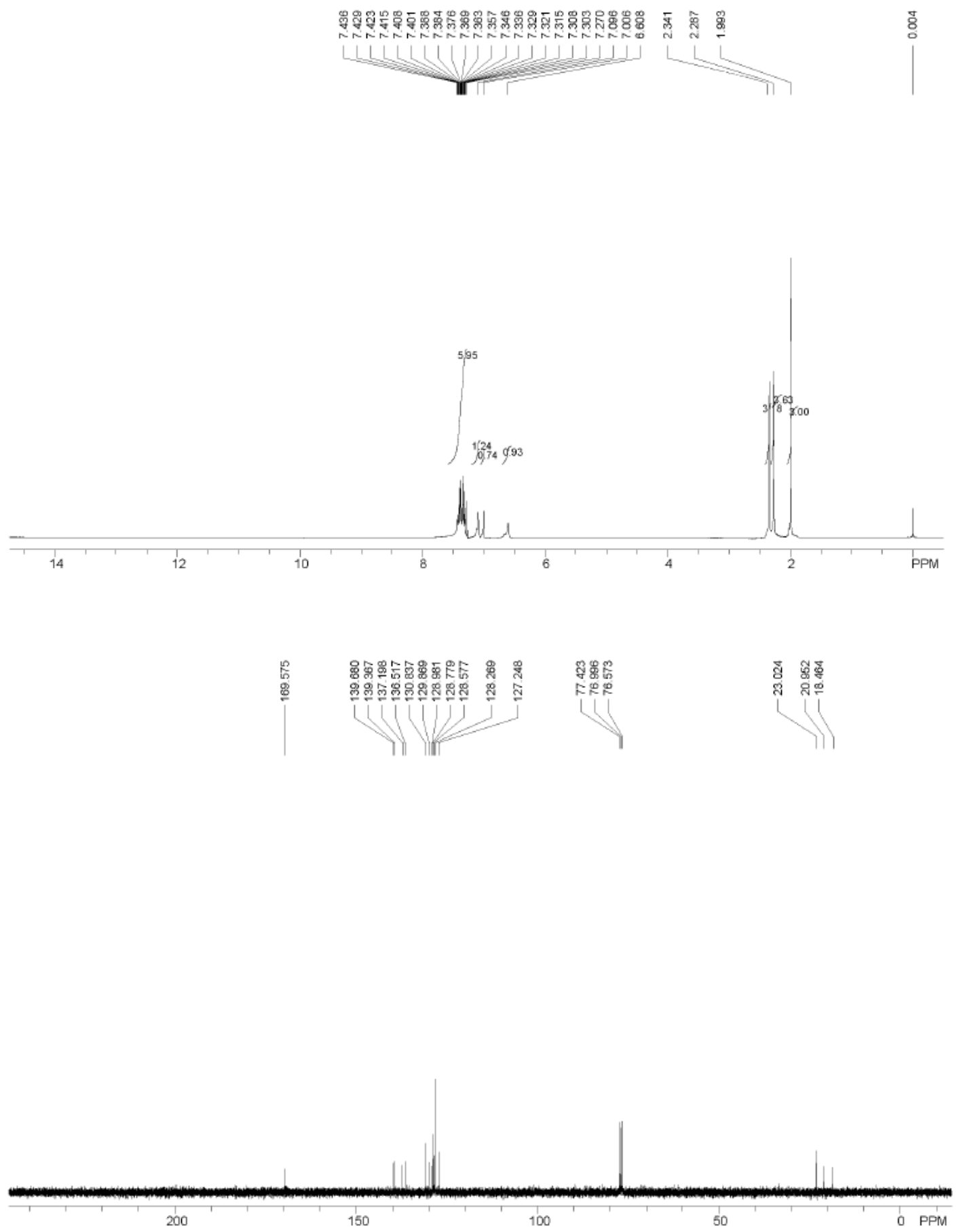

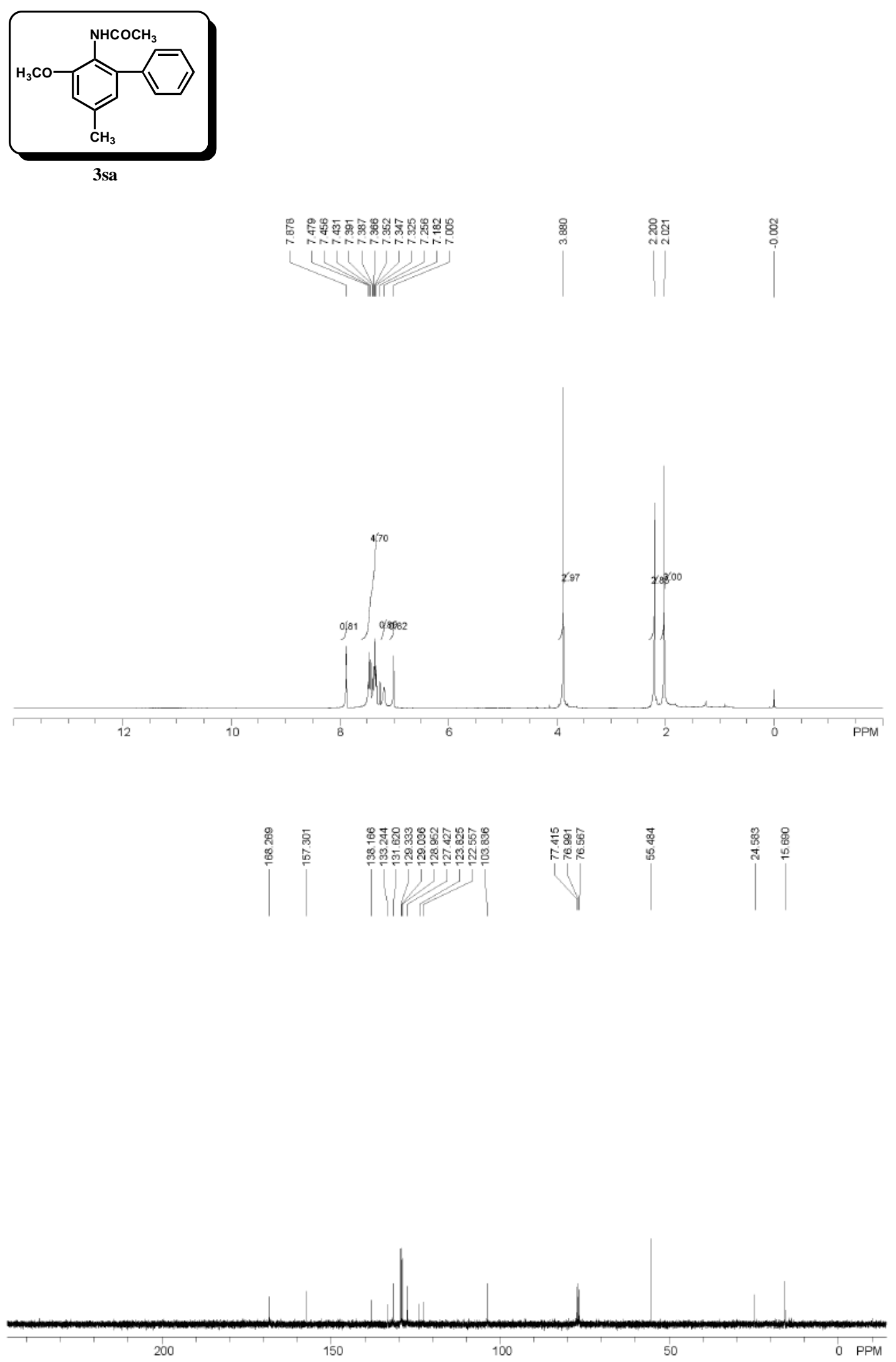

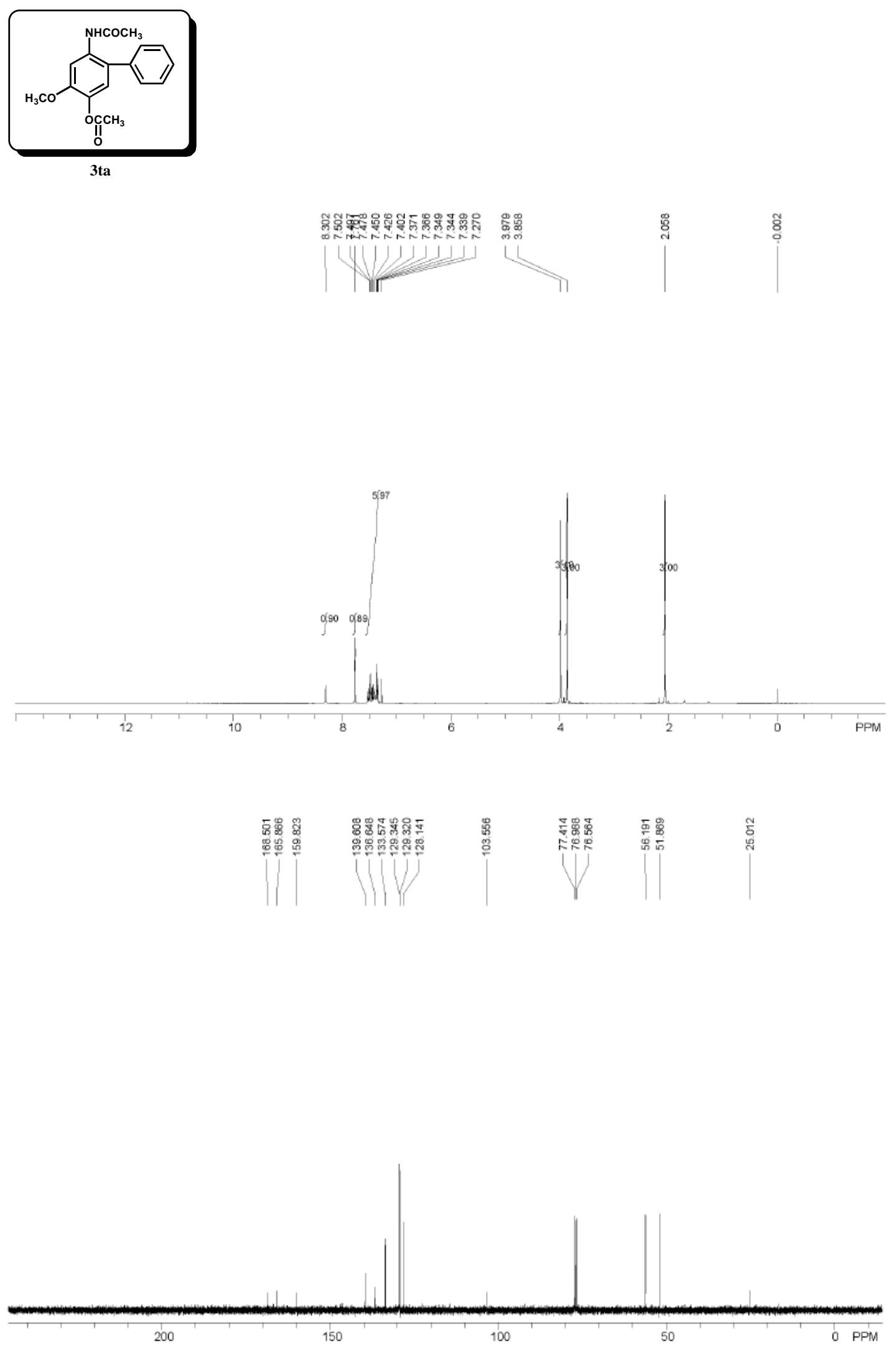